\title{
SUFFICIENT OPTIMALITY CRITERIA AND DUALITY FOR VARIATIONAL PROBLEMS WITH GENERALISED INVEXITY
}

\author{
B. MOND ${ }^{1}$ and I. HUSAIN ${ }^{2}$
}

(Received 4 February 1988; revised 16 December 1988)

\begin{abstract}
A number of Kuhn-Tucker type sufficient optimality criteria for a class of variational problems under weaker invexity assumptions are presented. As an application of these optimality results, various Mond-Weir type duality results are proved under a variety of generalised invexity assumptions. These results generalise many well-known duality results of variational problems and also give a dynamic analogue of certain corresponding (static) results relating to duality with generalised invexity in mathematical programming.
\end{abstract}

\section{Introduction}

Hanson [6] extended the Wolfe duality results of mathematical programming to a class of functions subsequently called invex. Since that time, it has been shown $[2,4,5,9]$ that many results in mathematical programming previously established for convex functions actually hold for the wider class of invex functions. Recently Mond, Chandra and Husain [8] extended the concept of invexity to continuous functions and used it to generalise earlier Wolfe duality results for a class of variational problems.

In [1] Bector, Chandra and Husain presented a dual to a variational problem in the spirit of Mond and Weir [11] different from that formulated by Mond and Hanson [10] in order to weaken the convexity conditions. In the present exposition, we present sufficient optimality conditions for variational problems under weaker convexity assumptions than those studied by Mond

\footnotetext{
'Department of Mathematics, La Trobe University, Bundoora, Melbourne, Victoria 3083.

${ }^{2}$ Department of Mathematics, Regional Engineering College, Hazratbal, India.

(C) Copyright Australian Mathematical Society 1989, Serial-fee code 0334-2700/89
} 
and Hanson. As an application of these criteria, we prove various Mond-Weir type duality theorems under a variety of generalised invexity conditions. We also establish a Mangasarian type strict converse duality theorem. It is also indicated that our duality theorems can be considered as dynamic generalisations of certain corresponding (static) duality theorems of mathematical programming.

\section{Notations and preliminaries}

Let $I=[a, b]$ be a real interval; let $f: I \times R^{n} \times R^{n} \rightarrow R$ be a continuously differentiable function. Consider $f(t, x(t), \dot{x}(t))$, where $x: I \rightarrow R^{n}$ is differentiable with derivative $\dot{x}$. Denote the partial derivative of $f$ by

$$
f_{t}, \quad f_{x}=\left[\frac{\partial f}{\partial x^{1}}, \frac{\partial f}{\partial x^{2}}, \ldots \frac{\partial f}{\partial x^{n}}\right], \quad f_{\dot{x}}=\left[\frac{\partial f}{\partial \dot{x}^{1}}, \frac{\partial f}{\partial \dot{x}^{2}}, \ldots \frac{\partial f}{\partial \dot{x}^{n}}\right] .
$$

Denote by $X$ the space of piecewise smooth functions $x: I \rightarrow R^{n}$, with the norm $\|x\|=\|x\|_{\infty}+\|D x\|_{\infty}$, where the differentiation operator $D$ is given by

$$
u=D x \Leftrightarrow x(t)=\alpha+\int_{a}^{t} u(s) d s,
$$

where $\alpha$ is a given boundary value; thus $D=\frac{d}{d t}$ except at discontinuities. For notational convenience $f(t, x(t), \dot{x}(t))$ will be written $f(t, x, \dot{x})$.

Let $F: X \rightarrow R$ defined by $F(X)=\int_{a}^{b} f(t, x(t), \dot{x}(t)) d t$ be Fréchet differentiable. In the subsequent analysis, no notational distinction is made between row and column vectors.

DeFINITION 1. Invex

The function $F$ is invex with respect to $\eta$ if there exists a differentiable vector function $\eta(t, x, \bar{x})$ with $\eta(t, x, x)=0$ such that for all $x, \bar{x} \in X$

$$
F(x)-F(\bar{x}) \geq \int_{a}^{b}\left\{\eta(t, x, \bar{x}) f_{x}(t, \bar{x}, \dot{x})+\left(\frac{d}{d t} \eta(t, x, \bar{x})\right) f_{\dot{x}}(t, \bar{x}, \dot{x})\right\} d t .
$$

Definition 2. Pseudoinvex (PIX)

The function $F$ is $P I X$ with respect to $\eta$ if there exists a differentiable vector function $\eta(t, x, \bar{x})$ with $\eta(t, x, x)=0$ such that for all $x, \bar{x} \in X$,

$$
\int_{a}^{b}\left\{\eta(t, x, \bar{x}) f_{x}(t, \bar{x}, \dot{x})+\left(\frac{d}{d t} \eta(t, x, \bar{x})\right) f_{\dot{x}}(t, \bar{x}, \dot{x})\right\} d t \geq 0 \Rightarrow F(x) \geq F(\bar{x})
$$

or equivalently

$$
\begin{aligned}
F(x) & <F(\bar{x}) \\
\Rightarrow & \int_{a}^{b}\left\{\eta(t, x, \bar{x}) f_{x}(t, \bar{x}, \dot{x})+\left(\frac{d}{d t} \eta(t, x, \bar{x})\right) f_{\dot{x}}(t, \bar{x}, \dot{x})\right\} d t<0 .
\end{aligned}
$$


Definition 3. Strictly Pseudoinvex (SPIX)

The functional $F$ is $S P I X$ with respect to $\eta$ if there exists a differentiable vector function $\eta(t, x, \bar{x})$ with $\eta(t, x, x)=0$ such that for all $x, \bar{x} \in X$,

$\int_{a}^{b}\left\{\eta(t, x, \bar{x}) f_{x}(t, \bar{x}, \dot{x})+\left(\frac{d}{d t} \eta(t, x, \bar{x})\right) f_{\dot{x}}(t, \bar{x}, \dot{x})\right\} d t \geq 0 \Rightarrow F(x)>F(\bar{x})$ or equivalently,

$$
F(x) \leq F(\bar{x}) \Rightarrow \int_{a}^{b}\left\{\eta(t, x, \bar{x}) f_{x}(t, \bar{x}, \dot{x})+\left(\frac{d}{d t} \eta(t, x, \bar{x})\right) f_{\dot{x}}(t, \bar{x}, \dot{x})\right\} d t<0
$$

DefinITION 4. Quasi-invex ( $Q I X)$

The functional $F$ is $Q I X$ with respect to $\eta$ if there exists a differentiable vector function $\eta(t, x, x)$ with $\eta(t, x, x)=0$ such that

$$
\int_{a}^{b}\left\{\eta(t, x, \bar{x}) f_{x}(t, \bar{x}, \dot{x})+\left(\frac{d}{d t} \eta(t, x, \tilde{x})\right) f_{\dot{x}}(t, \bar{x}, \dot{x})\right\} d t>0 \Rightarrow F(x)>F(\bar{x})
$$

or equivalently,

$$
F(x) \leq F(\bar{x}) \Rightarrow \int_{a}^{b}\left\{\eta(t, x, \bar{x}) f_{x}(t, \bar{x}, \dot{x})+\left(\frac{d}{d t} \eta(t, x, \bar{x})\right) f_{\dot{x}}(t, \bar{x}, \dot{x})\right\} d t \leq 0 .
$$

In the above definitions, $d \eta / d t$ is the vector whose $i$ th component is $(d / d t) \eta^{i}(t, x, \bar{x})$. It is to be noted here that these definitions are very much in the spirit of Hanson [6] and Bector, Chandra and Husain [1]. Here if $f$ is independent of $t$, definitions (1-4) reduce to the definitions of invexity, pseudoinvexity, strict-pseudo invexity and quasi-invexity of the static case [6] respectively. In [6] the author does not require $\eta(x, x)=0$, but this is usually satisfied in most examples for $\eta$ (see $[2,5]$ ). As is known $[2,4,5]$, pseudoinvexity, strict-pseudo-invexity and quasi-invexity are generalisations of pseudoconvexity, strict pseudo-convexity and quasiconvexity respectively.

Now consider the determination of a piecewise smooth extremal $x=x(t)$, $a \leq t \leq b$, for the following problem:

Problem I. (Primal) $\equiv P$

$$
\begin{aligned}
& \underset{x \in X}{\operatorname{Minimise}} \phi(x)=\int_{a}^{b} f(t, x(t), \dot{x}(t)) d t \\
& \text { subject to } \\
& \qquad x(a)=\alpha, \quad x(b)=\beta
\end{aligned}
$$

and

$$
g(t, x(t), \dot{x}(t)) \leq 0, \quad t \in I
$$


Consider also the determination of an $m+n$ dimensional extremal $(u, y)=$ $(u(t), y(t)), t \in I$, for the following maximisation problem:

Problem II. (Dual) $\equiv D$

Maximise $\psi(u, y)=\int_{a}^{b}\{f(t, u, \dot{u})+y(t) g(t, u, \dot{u})\} d t$

subject to

$$
\begin{gathered}
u(a)=\alpha, \quad u(b)=\beta \\
f_{u}(t, u, \dot{u})+y(t) g_{u}(g, u, \dot{u})=\frac{d}{d t}\left\{f_{\dot{u}}(t, u, \dot{u})+y(t) g_{\dot{u}}(t, u, \dot{u})\right\} \\
y(t) \geq 0, \quad t \in I
\end{gathered}
$$

Here $u(t)$ is an $n$-dimensional piecewise smooth function and $y(t)$ is an $m$-dimensional function continuous except possibly for the values of $t$ corresponding to corners of $u(t)$. For values of $t$ corresponding to corners of $u(t)$, (6) must be satisfied for right and left hand limits. The equation (6) means

$$
\begin{aligned}
f_{u^{1}}+\left(\sum_{i=1}^{m} y^{i}(t) g^{i}\right)_{u^{1}}= & \frac{d}{d t}\left\{f_{\dot{u}^{1}}+\left(\sum_{i=1}^{m} y^{i}(t) g^{i}\right)_{\dot{u}^{1}}\right\}, \\
f_{u^{2}}+\left(\sum_{i=1}^{m} y^{i}(t) g^{i}\right)_{u^{2}}= & \frac{d}{d t}\left\{f_{\dot{u}^{2}}+\left(\sum_{i=1}^{m} y^{i}(t) g^{i}\right)_{\dot{u}^{2}}\right\}, \\
& \vdots \\
f_{u^{n}}+\left(\sum_{i=1}^{m} y^{i}(t) g^{i}\right)_{u^{n}}= & \frac{d}{d t}\left\{f_{\dot{u}^{n}}+\left(\sum_{i=1}^{m} y^{i}(t) g^{i}\right)_{\dot{u}^{n}}\right\} .
\end{aligned}
$$

Mond, Chandra and Husain [8] have established the following results.

THEOREM 1 (Weak Duality). If $f$ and $g$ are invex for some function $\eta$, then the infimum of $(P)$ is greater than or equal to the supremum of $(D)$.

THEOREM 2 (Strong Duality). Assume that an appropriate constraint qualification is satisfied and that $f$ and $g$ are invex for the same function $\eta$. If the function $x^{*}(t)$ minimises the primal problem $(P)$, then there exists a $y^{*}(t)$ such that $\left(x^{*}(t), y^{*}(t)\right)$ maximises the dual problem $(D)$ and the extreme values of $(P)$ and $(D)$ are equal.

Let $H$ denote the set of all feasible solutions of the problem $(P)$; that is, let $H=\{x \in X \mid g(t, x(t), \dot{x}(t)) \leq 0, t \in I\}$. 
For any $x^{*} \in H$, let $M\left(x^{*}\right)$ denote the index set of all the binding inequality constraints at $\left(x^{*}(t), \dot{x}^{*}(t)\right)$; that is, let

$$
M\left(x^{*}\right)=\left\{i \in\{1,2,3, \ldots m\} \mid g^{i}\left(t, x^{*}(t), \dot{x}^{*}(t)\right)=0, t \in I\right\} .
$$

Let $g^{M\left(x^{*}\right)}$ denote the vector function having components $g^{l}$ with $i \in$ $M\left(x^{*}\right)$.

\section{Kuhn-Tucker type sufficient optimality theorems}

In this section, we present some sufficient optimality criteria of the KuhnTucker type for the problem $(P)$.

THeORem 3. Let $x^{*} \in H$ and assume that $\phi$ is PIX at $x^{*}$ with respect to $\eta$ and that for each $i \in M\left(x^{*}\right), g^{i}$ is $Q I X$ at $x^{*}$ with respect to $\eta$. If there exists a piecewise smooth $y^{*}: I \rightarrow R^{m}$ such that $\left(x^{*}(t), y^{*}(t)\right)$ satisfies the conditions

$$
\begin{gathered}
f_{x}\left(t, x^{*}, \dot{x}^{*}\right)+y^{*}(t) g_{x}\left(t, x^{*}, \dot{x}^{*}\right)=\frac{d}{d t}\left\{f_{\dot{x}}\left(t, x^{*}, \dot{x}^{*}\right)+y^{*}(t) g_{\dot{x}}\left(t, x^{*}, \dot{x}^{*}\right)\right\} \\
y^{*}(t) g\left(t, x^{*}(t), \dot{x}^{*}(t)\right)=0, \quad t \in I \\
y^{*}(t) \geq 0, \quad t \in I,
\end{gathered}
$$

then $x^{*}$ is a global optimal solution of Problem $(P)$.

Proof. Since for any $x \in X$,

$$
g^{M\left(x^{*}\right)}(t, x(t), \dot{x}(t)) \leq 0=g^{M\left(x^{*}\right)}\left(t, x^{*}(t), \dot{x}^{*}(t)\right), \quad t \in I,
$$

by the quasi-invexity assumption, we have that for $t \in I, i \in M\left(x^{*}\right)$,

$$
\eta\left(t, x, x^{*}\right) g_{x}^{i}\left(t, x^{*}, \dot{x}^{*}\right)+\left(\frac{d}{d t} \eta\left(t, x, x^{*}\right)\right) g_{\dot{x}}^{i}\left(t, x^{*}, \dot{x}^{*}\right) \leq 0,
$$

and hence, by taking $y^{i^{*}}(t)=0$ for $i \notin M\left(x^{*}\right)$,

$$
\begin{aligned}
& \int_{a}^{b}\left\{\eta\left(t, x, x^{*}\right) y^{*}(t) g_{x}\left(t, x^{*}, \dot{x}^{*}\right)\right. \\
& \left.\quad+\left(\frac{d}{d t} \eta\left(t, x, x^{*}\right)\right) y^{*}(t) g_{\dot{x}}\left(t, x^{*}, \dot{x}^{*}\right)\right\} d t \leq 0, \text { for all } x \in H .
\end{aligned}
$$


From (8), we have

$$
\begin{aligned}
\int_{a}^{b} & \eta\left(t, x, x^{*}\right)\left\{f_{x}\left(t, x^{*}, \dot{x}^{*}\right)+y^{*}(t) g_{x}\left(t, x^{*}, \dot{x}^{*}\right)\right\} d t \\
& =\int_{a}^{b} \eta\left(t, x, x^{*}\right) \frac{d}{d t}\left(f_{\dot{x}}\left(t, x^{*}, \dot{x}^{*}\right)+y^{*}(t) g_{\dot{x}}\left(t, x^{*}, \dot{x}^{*}\right)\right) d t \\
= & \left.\eta\left(t, x, x^{*}\right)\left(f_{\dot{x}}\left(t, x^{*}, \dot{x}^{*}\right)+y^{*}(t) g_{\dot{x}}\left(t, x^{*}, \dot{x}^{*}\right)\right)\right|_{t=a} ^{t=b} \\
& -\int_{a}^{b} \frac{d}{d t} \eta\left(t, x, x^{*}\right)\left(f_{\dot{x}}\left(t, x^{*}, \dot{x}^{*}\right)+y^{*}(t) g_{\dot{x}}\left(t, x^{*}, \dot{x}^{*}\right)\right) d t,
\end{aligned}
$$

(integrating by parts).

Thus,

$$
\begin{aligned}
& \int_{a}^{b} \eta\left(t, x, x^{*}\right)\left\{f_{x}\left(t, x^{*}, \dot{x}^{*}\right)+y^{*}(t) g_{x}\left(t, x^{*}, \dot{x}^{*}\right)\right\} d t \\
& \quad+\int_{a}^{b} \frac{d}{d t} \eta\left(t, x, x^{*}\right)\left(f_{\dot{x}}\left(t, x^{*}, \dot{x}^{*}\right)+y^{*}(t) g_{\dot{x}}\left(t, x^{*}, \dot{x}^{*}\right)\right) d t=0
\end{aligned}
$$

(using (2), (5) and $\eta(t, x, x)=0$ ). From (12), we have

$$
\begin{gathered}
\int_{a}^{b}\left\{\eta\left(t, x, x^{*}\right) f_{x}\left(t, x^{*}, \dot{x}^{*}\right)+\left(\frac{d}{d t} \eta\left(t, x, x^{*}\right)\right) f_{\dot{x}}\left(t, x^{*}, \dot{x}^{*}\right)\right\} d t \\
=-\int_{a}^{b}\left\{\eta\left(t, x, x^{*}\right) y^{*}(t) g_{x}\left(t, x^{*}, \dot{x}^{*}\right)\right. \\
\left.+\left(\frac{d}{d t} \eta\left(t, x, x^{*}\right)\right) y^{*}(t) g_{\dot{x}}\left(t, x^{*}, \dot{x}^{*}\right)\right\} d t
\end{gathered}
$$

From (13) and (11), we have

$$
\int_{a}^{b}\left\{\eta\left(t, x, x^{*}\right) f_{x}\left(t, x^{*}, \dot{x}^{*}\right)+\left(\frac{d}{d t} \eta\left(t, x, x^{*}\right)\right) f_{\dot{x}}\left(t, x^{*}, \dot{x}^{*}\right)\right\} d t \geq 0
$$

Now (14) in view of the pseudoinvexity of $\phi$ yields

$$
\int_{a}^{b} f(t, x, \dot{x}) d t \geq \int_{a}^{b} f\left(t, x^{*}, \dot{x}^{*}\right) d t, \forall x \in H
$$

that is, $x^{*}$ is a global optimal solution of $(P)$.

The next two theorems show that global optimality is maintained if, instead of the individual constraint function $g^{i}$, a certain function, defined in terms of $g^{i}$, has an appropriate weak invexity property.

THEOREM 4. Let $x^{*} \in H$ and suppose that $\phi$ is pseudoconvex at $x^{*}$ with respect to $\eta$. If there exists a piecewise smooth $y^{*}: I \rightarrow R^{m}$ such tht $\left(x^{*}, y^{*}\right)$ satisfies (8) $-(10)$ and if the function $\theta\left(\cdot, y^{*}\right): X \rightarrow R$ defined by

$$
\boldsymbol{\theta}\left(x, y^{*}\right)=\int_{a}^{b} y^{*}(t) g(t, x(t), \dot{x}(t)) d t
$$


is QIX at $x^{*}$, with respect to $\eta$, then $x^{*}$ is a global optimal solution of the problem $(P)$.

Proof. For any $x \in X$,

$$
\int_{a}^{b} y^{*}(t) g(t, x(t), \dot{x}(t)) d t \leq 0=\int_{a}^{b} y^{*}(t) g\left(t, x^{*}(t), \dot{x}^{*}(t)\right) d t
$$

which, in view of the quasi-invexity of $\Theta\left(\cdot, y^{*}\right)$ implies for all $x \in H$

$$
\begin{aligned}
& \int_{a}^{b}\left\{\eta\left(t, x, x^{*}\right) y^{*}(t) g_{x}\left(t, x^{*}(t), \dot{x}^{*}(t)\right)\right. \\
& \left.\quad+\left(\frac{d}{d t} \eta\left(t, x, x^{*}\right)\right) y^{*}(t) g_{\dot{x}}\left(t, x^{*}(t), \dot{x}^{*}(t)\right)\right\} d t \leq 0 .
\end{aligned}
$$

This is just (11) which, as in the proof of the previous theorem, implies $\int_{a}^{b}\left\{\eta\left(t, x, x^{*}\right) f_{x}\left(t, x^{*}, \dot{x}^{*}\right)+\left(\frac{d}{d t} \eta\left(t, x, x^{*}\right)\right) f_{\dot{x}}\left(t, x^{*}, \dot{x}^{*}\right)\right\} d t \geq 0, \forall x \in H$ which, by pseudoinvexity of $\phi$ at $x^{*}$ (with respect to $H$ ), yields

$$
\int_{a}^{b} f\left(t, x^{*}, \dot{x}^{*}\right) d t \leq \int_{a}^{b} f(t, x, \dot{x}) d t, \forall x \in H .
$$

Thus $x^{*}$ is a global optimal solution of $(P)$.

THeorem 5. Let $x^{*} \in H$ and suppose that $\phi$ is $Q I X$ at $x^{*}$ with respect to $\eta$. If there exists a piecewise smooth function $y^{*}: I \rightarrow R^{m}$ such that $(8)-(10)$ are satisfied and if the function $\Theta\left(\cdot, y^{*}\right)$ is SPIX at $x^{*}$ with respect to $\eta$, then $x^{*}$ is a global optimal solution of the problem $(P)$.

Proof. In view of strict-pseudoinvexity of $\Theta\left(\cdot, y^{*}\right)$, from (15), it follows that

$$
\begin{aligned}
& \int_{a}^{b}\left\{\eta\left(t, x, x^{*}\right) y^{*}(t) g\left(t, x^{*}, \dot{x}^{*}\right)\right. \\
& \left.\quad+\left(\frac{d}{d t} \eta\left(t, x, x^{*}\right)\right) y^{*}(t) g_{\dot{x}}\left(t, x^{*}, \dot{x}^{*}\right)\right\} d t<0, \forall x \in H
\end{aligned}
$$

which, in a manner analogous to the proof of the previous two theorems, implies

$$
\int_{a}^{b}\left\{\eta\left(t, x, x^{*}\right) f_{x}\left(t, x^{*}, \dot{x}^{*}\right)+\left(\frac{d}{d t} \eta\left(t, x, x^{*}\right)\right) f_{\dot{x}}\left(t, x^{*}, \dot{x}^{*}\right)\right\} d t>0, \forall x \in H .
$$

This inequality in view of the quasi-invexity of $\phi$ at $x^{*}$ implies

$$
\int_{a}^{b} f\left(t, x^{*}, \dot{x}^{*}\right) d t \leq \int_{a}^{b} f(t, x, \dot{x}) d t, \forall x \in H .
$$

Lastly we formulate a global criterion in which only the Lagrangian function associated with $(P)$ is assumed to be pseudoinvex. 
THEOREM 6. Let $x^{*} \in H$. If there exists a piecewise smooth $y^{*}: I \rightarrow R^{m}$ such that $\left(x^{*}(t), y^{*}(t)\right)$ satisfies (8)-(10), and if the Lagrangian function $\psi\left(x, y^{*}\right)$ : $X \rightarrow R$, defined by

$$
\psi\left(x, y^{*}\right)=\int_{a}^{b}\left\{f(t, x, \dot{x})+y^{*}(t) g(t, x, \dot{x})\right\} d t
$$

is PIX at $x^{*}$ with respect to $\eta$, then $x^{*}$ is a global optimal solution of $(P)$.

Proof. The equation (8) yields (13), i.e.,

$$
\begin{aligned}
& \int_{a}^{b}\left\{\eta\left(t, x, x^{*}\right)\left(f_{x}\left(t, x^{*}, \dot{x}^{*}\right)+y^{*}(t) g_{x}\left(t, x^{*}, \dot{x}^{*}\right)\right)\right. \\
& \left.\quad+\frac{d}{d t} \eta\left(t, x, x^{*}\right)\left(f_{\dot{x}}\left(t, x^{*}, \dot{x}^{*}\right)+y^{*}(t) g_{\dot{x}}\left(t, x^{*}, \dot{x}^{*}\right)\right)\right\} d t=0, \forall x \in H,
\end{aligned}
$$

which, by the pseudo-invexity assumption for $\psi\left(\cdot, y^{*}\right)$ gives

$$
\psi\left(x^{*}, y^{*}\right) \leq \psi\left(x, y^{*}\right), \forall x \in H,
$$

that is,

$$
\int_{a}^{b}\left\{f\left(t, x^{*}, \dot{x}^{*}\right)+y^{*}(t) g\left(t, x^{*}, \dot{x}^{*}\right)\right\} d t \leq \int_{a}^{b}\left\{f(t, x, \dot{x})+y^{*}(t) g(t, x, \dot{x})\right\} d t .
$$

By (3), (9) and (10), this inequality leads to

$$
\int_{a}^{b} f\left(t, x^{*}, \dot{x}^{*}\right) d t \leq \int_{a}^{b} f(t, x, \dot{x}) d t, \forall x \in H,
$$

which means that $x^{*}$ is a global optimal solution of $(P)$.

REMARK. Although not explicitly stated, in the last four theorems, the stated invexity and weakened invexity requirements need only hold at $x^{*}$ for all feasible $x$ of $(P)$.

\section{Mond-Weir type duality}

We now establish duality between the problem $(P)$ and the following Mond-Weit type dual $\left(D_{0}\right)$, formulated by Bector, Chandra and Husain [1]. Problem III. (Dual) $\equiv D_{0}$

$$
\begin{aligned}
& \text { Maximise } \int_{a}^{b} f(t, u(t), \dot{u}(t)) d t \\
& \text { subject to } \\
& \qquad u(a)=\alpha, \quad u(b)=\beta,
\end{aligned}
$$




$$
\begin{gathered}
f_{u}(t, u, \dot{u})+y(t) g_{u}(t, u, \dot{u})=\frac{d}{d t}\left\{f_{\dot{u}}(t, u, \dot{u})+y(t) g_{\dot{u}}(t, u, \dot{u})\right\} \\
\int_{a}^{b} y(t) g(t, u, \dot{u}) d t \geq 0 \\
y(t) \geq 0, \quad t \in I .
\end{gathered}
$$

THEOREM 7 (Weak Duality). If for all $x \in X$ and $(u, y) \in Z$, the feasible set of the dual $\left(D_{0}\right)$, there exists a differentiable vector function $\eta$ with $\eta(t, x, x)=$ 0 such that, for all feasible $(x, u, y)$

(I) $\phi$ and $\theta(\cdot, y)$ are respectively $P I X$ and $Q I X$ at $u$, or

(II) $\phi$ and $\theta(\cdot, y)$ are respectively $Q I X$ and SPIX at $u$,

with respect to the same $\eta$, then $\int_{a}^{b} f(t, x, \dot{x}) d t \geq \int_{a}^{b} f(t, u, \dot{u}) d t$.

Proof. Since $x \in H$ and $(u, y) \in Z$, we have, from (3), (19) and (18),

$$
\int_{a}^{b} y(t) g(t, x, \dot{x}) d t \leq \int_{a}^{b} y(t) g(t, u, \dot{u}) d t .
$$

(I) The inequality (20) and quasi-invexity of $\theta(\cdot, y)$ imply

$$
\int_{a}^{b}\left\{\eta(t, x, u) y(t) g_{u}(t, u, \dot{u})+\left(\frac{d}{d t} \eta(t, x, u)\right) y(t) g_{\dot{u}}(t, u, \dot{u})\right\} d t \leq 0 \text {. }
$$

The constraint (17), as earlier, is equivalent to

$$
\begin{aligned}
\int_{a}^{b} & \left\{\eta(t, x, u) f_{u}(t, u, \dot{u})+\left(\frac{d}{d t} \eta(t, x, u)\right) f_{\dot{u}}(t, u, \dot{u})\right\} d t \\
& +\int_{a}^{b}\left\{\eta(t, x, u) y(t) g_{u}(t, u, \dot{u})+\left(\frac{d}{d t} \eta(t, x, u)\right) y(t) g_{\dot{u}}(t, u, \dot{u})\right\} d t=0
\end{aligned}
$$

that is,

$$
\begin{aligned}
& \int_{a}^{b}\left\{\eta(t, x, u) f_{u}(t, u, \dot{u})+\left(\frac{d}{d t} \eta(t, x, u)\right) f_{\dot{u}}(t, u, \dot{u})\right\} d t \\
& \quad=-\int_{a}^{b}\left\{\eta(t, x, u) y(t) g_{u}(t, u, \dot{u})+\left(\frac{d}{d t} \eta(t, x, u)\right) y(t) g_{\dot{u}}(t, u, \dot{u})\right\} d t
\end{aligned}
$$

Using (21), this inequality yields

$$
\int_{a}^{b}\left\{\eta(t, x, u) f_{u}(t, u, \dot{u})+\left(\frac{d}{d t} \eta(t, x, u)\right) f_{\dot{u}}(t, u, \dot{u})\right\} d t \geq 0
$$

This inequality along with pseudo-invexity of $\phi$ yields

$$
\int_{a}^{b} f(t, x, \dot{x}) d t \geq \int_{a}^{b} f(t, u, \dot{u}) d t
$$


(II) The inequality (20), in view of strict-pseudo invexity of $\Theta(\cdot, y)$ implies

$\int_{a}^{b}\left\{\eta(t, x, u) y(t) g_{u}(t, u, \dot{u})+\left(\frac{d}{d t} \eta(t, x, u)\right) y(t) g_{\dot{u}}(t, u, \dot{u})\right\} d t<0$.

Now (17) along with (24) yields

$$
\int_{a}^{b}\left\{\eta(t, x, u) f_{u}(t, u, \dot{u})+\left(\frac{d}{d t} \eta(t, x, u)\right) f_{\dot{u}}(t, u, \dot{u})\right\} d t>0,
$$

which, in view of the quasi-invexity of $\phi$ implies

$$
\int_{a}^{b} f(t, x, \dot{x}) d t \geq \int_{a}^{b} f(t, u, \dot{u}) d t
$$

TheOREM 8 (Strong Duality). Let $x^{*}$ be a normal $[1,10]$ solution of $(P)$. Assume that a differentiable vector function $\eta$, with $\eta(t, x, x)=0$, exists such that the hypotheses of Theorem 7 are satisfied. Then there exists a piecewise smooth $y^{*}: I \rightarrow R^{m}$ such that $\left(x^{*}, y^{*}\right)$ solves $\left(D_{0}\right)$ and

$$
\operatorname{Minimum}(P)=\operatorname{Maximum}\left(D_{0}\right) \text {. }
$$

Proof. Since $x^{*}$ solves $(P)$ and is normal $[1,10]$, from Valentine necessary conditions [12] or Kuhn-Tucker type optimality conditions [3] it follows that there exists a piecewise $y^{*}: I \rightarrow R^{m}$ such that $\left(x^{*}, y^{*}\right)$ satisfies

$$
\begin{gathered}
f_{x}\left(t, x^{*}, \dot{x}^{*}\right)+y^{*}(t) g_{x}\left(t, x^{*}, \dot{x}^{*}\right)=\frac{d}{d t}\left\{f_{\dot{x}}\left(t, x^{*}, \dot{x}^{*}\right)+y^{*}(t) g_{\dot{x}}\left(t, x^{*}, \dot{x}^{*}\right)\right\}, \\
\int_{a}^{b} y^{*}(t) g\left(t, x^{*}, \dot{x}^{*}\right) d t=0, \\
y(t) \geq 0, \quad t \in I .
\end{gathered}
$$

Thus, from the above relations, it follows that $\left(x^{*}, y^{*}\right) \in Z$.

Obviously, the objective functionals of $(P)$ and $\left(D_{0}\right)$ are equal, so the value of $(P)$ equals the value of $\left(D_{0}\right)$ at $x^{*}$. Optimality follows by invoking weak duality, Theorem 7 .

THEOREM 9 (Strict Converse Duality). Let $x^{*}$ be an optimal solution of $(P)$ and be normal $[1,10]$. Assume that there exists a nondifferentiable vector function $\eta$ with $\eta(t, x, x)=0$ such that the hypotheses of Theorem 7 hold for all $x \in H$ and $(u, y) \in Z$. If $(\bar{u}, \bar{y})$ is an optimal solution of $\left(D_{0}\right)$ and, with respect to $\eta$ at $\bar{u}$,

(i) $\psi(\cdot, y)$ is SPIX, or

(ii) $\phi$ is $S P I X$ and $\Theta(\cdot, y)$ is $Q I X$, or 
(iii) $\phi$ is QIX and $\boldsymbol{\theta}(\cdot, y)$ is SPIX,

then $\bar{u}=x^{*}$, that is $\bar{u}$ is a global optimal solution of $(P)$.

Proof. Suppose that $x^{*} \neq \vec{u}$. Since $x^{*}$ is an optimal solution of $(P)$ and is normal, it follows from Theorem 8 that there exists a piecewise smooth $\hat{y}: I \rightarrow R^{m}$ such that $\left(x^{*}, \hat{y}\right)$ is an optimal solution of $\left(D_{0}\right)$. Since $(\bar{u}, \bar{y})$ is also an optimal solution of $\left(D_{0}\right)$, it follows that

$$
\begin{gathered}
\int_{a}^{b} f\left(t, x^{*}, \dot{x}^{*}\right) d t=\int_{a}^{b} f(t, \bar{u}, \dot{u}) d t \\
\int_{a}^{b} \bar{y}(t) g(t, \bar{u}, \dot{\bar{u}}) d t \geq 0 .
\end{gathered}
$$

(i) The equation (17) is equivalent to

$$
\begin{aligned}
& \int_{a}^{b}\left(\eta\left(t, x^{*}, \bar{u}\right)\left\{f_{u}(t, \bar{u}, \dot{u})+\bar{y}(t) g_{u}(t, \tilde{u}, \dot{u})\right\}\right. \\
& \quad+\frac{d}{d t} \eta\left(t, x^{*}, \vec{u}\right)\left\{f_{\dot{u}}(t, \tilde{u}, \dot{u})+\bar{y}(t) g_{\dot{u}}(t, \vec{u}, \dot{u}\}\right) d t=0 .
\end{aligned}
$$

This, with strict-pseudo-invexity of $\psi(\cdot, \bar{y})$ implies

$$
\int_{a}^{b}\left\{f\left(t, x^{*}, \dot{x}^{*}\right)+\bar{y}(t) g\left(t, x^{*}, \dot{x}^{*}\right)\right\} d t>\int_{a}^{b}\{f(t, \bar{u}, \dot{\bar{u}})+\bar{y}(t) g(t, \bar{u}, \dot{u})\} d t .
$$

From $\tilde{y}(t) \geq 0, t \in I$ and $g\left(t, x^{*}, \dot{x}^{*}\right) \leq 0, t \in I$, we get

$$
\int_{a}^{b} \tilde{y}(t) g\left(t, x^{*}, \dot{x}^{*}\right) d t \leq 0 .
$$

Now (25), (26) and (28) contradict (29). Hence $x^{*}(t)=\bar{u}(t), t \in I$.

(ii) For all $x \in X$ and $(u, y) \in Z$,

$$
\int_{a}^{b} y(t) g(t, x, \dot{x}) d t \leq \int_{a}^{b} y(t) g(t, u, \dot{u}) d t
$$

and, by quasi-invexity of $\boldsymbol{\theta}(\cdot, y)$ at $u$, we have

$$
\int_{a}^{b}\left\{\eta(t, x, u) y(t) g_{u}(t, u, \dot{u})+\left(\frac{d}{d t} \eta(t, x, u)\right) y(t) g_{\dot{u}}(t, u, \dot{u})\right\} d t \leq 0 .
$$

The inequality (22) along with this yields

$$
\int_{a}^{b}\left\{\eta(t, x, u) f_{u}(t, u, \dot{u})+\left(\frac{d}{d t} \eta(t, x, u)\right) f_{\dot{u}}(t, u, \dot{u})\right\} d t \geq 0
$$


From (31) and strict-pseudo invexity of $\phi$ at $\bar{u}$, we have

$$
\begin{gathered}
\int_{a}^{b}\left\{\eta(t, x, \bar{u}) f_{u}(t, \bar{u}, \dot{u})+\left(\frac{d}{d t} \eta(t, x, \bar{u})\right) f_{\dot{u}}(t, \bar{u}, \dot{u})\right\} d t \geq 0 \\
\Rightarrow \int_{a}^{b} f(t, x, \dot{x}) d t>\int_{a}^{b} f(t, \bar{u}, \dot{u}) d t, \text { for all } x \in H,
\end{gathered}
$$

and since $x^{*} \in H$, we have

$$
\int_{a}^{b} f\left(t, x^{*}, \dot{x}^{*}\right) d t>\int_{a}^{b} f(t, \bar{u}, \dot{u}) d t
$$

contradicting (25). Hence $x^{*}(t)=\bar{u}(t), t \subset I$.

(iii) From (30) along with strict-pseudo invexity of $\Theta(\cdot, y)$, we have

$$
\int_{a}^{b}\left\{\eta(t, x, u) y(t) g_{u}(t, u, \dot{u})+\left(\frac{d}{d t} \eta(t, x, u)\right) y(t) g_{\dot{u}}(t, u, \dot{u})\right\} d t<0
$$

This inequality, along with (22), yields

$$
\int_{a}^{b}\left\{\eta(t, x, u) f_{u}(t, u, \dot{u})+\left(\frac{d}{d t} \eta(t, x, u)\right) f_{\dot{u}}(t, u, \dot{u})\right\} d t>0 .
$$

In view of the quasi-invexity of $\phi$ at $\bar{u}$,

$$
\begin{gathered}
\int_{a}^{b}\left\{\eta(t, x, \bar{u}) f_{u}(t, \bar{u}, \dot{u})+\left(\frac{d}{d t} \eta(t, x, \bar{u})\right) f_{\dot{u}}(t, \bar{u}, \dot{u})\right\} d t>0 \\
\Rightarrow \int_{a}^{b} f(t, x, \dot{x}) d t>\int_{a}^{b} f(t, \tilde{u}, \dot{u}) d t, \text { for all } x \in H .
\end{gathered}
$$

As in (ii), we have

$$
\int_{a}^{b} f\left(t, x^{*}, \dot{x}^{*}\right) d t>\int_{a}^{b} f(t, \tilde{u}, \dot{u}) d t
$$

a contradiction to (25).

\section{Related problems}

The duality results here can be extended to corresponding variational problems with natural boundary values rather than fixed end points.

Primal $\left(P_{1}\right)$ : Minimise $\int_{a}^{b} f(t, x, \dot{x}) d t$

$$
\text { subject to } g(t, x, \dot{x}) \leq 0, \quad t \in I \text {. }
$$


Dual $\left(D_{1}\right)$ : Maximise $\int_{a}^{b} f(t, u, \dot{u}) d t$ subject to

$$
\begin{gathered}
f_{u}(t, u, \dot{u})+y(t) g_{u}(t, u, \dot{u})=\frac{d}{d t}\left\{f_{\dot{u}}(t, u, \dot{u})+y(t) g_{\dot{u}}(t, u, \dot{u})\right\}, \\
\int_{a}^{b} y(t) g(t, u, \dot{u}) d t \geq 0, \quad y(t) \geq 0, t \in I, \\
f_{\dot{u}}(t, u, \dot{u})+\left.y(t) g_{\dot{u}}(t, u, \dot{u})\right|_{t=a}=0, \\
f_{\dot{u}}(t, u, \dot{u})+\left.y(t) g_{\dot{u}}(t, u, \dot{u})\right|_{t=b}=0 .
\end{gathered}
$$

By $f_{\dot{u}}(t, u, \dot{u})+\left.y(t) g_{\dot{u}}(t, u, \dot{u})\right|_{t=a, b}$, we mean that $f_{\dot{u}}(t, u, \dot{u})+y(t) g_{\dot{u}}(t, u, \dot{u})$ is evaluated at $t=a$ and $t=b$.

We shall not repeat the proof of Theorems 3-10 but merely point out the modification in the arguments that are required for the theorems to remain valid. In the proofs of theorems in the preceding sections, (2), (5) or (16) and $\eta(t, x, x)=0$ were utilised to guarantee that the integrated part $\left.\eta(t, x, u)\left(f_{\dot{u}}+y(t) g_{\dot{u}}\right)\right|_{t=a} ^{t=b}$ is zero. It is obvious that even though (2) and (16) are lacking in the problems with natural boundary values, the terms $\left.\eta(t, x, u)\left(f_{\dot{u}}+y(t) g_{\dot{u}}\right)\right|_{t=a} ^{t=b}$ still vanish by virtue of (32) and (33). If only one end point is fixed, say $x(a)=\alpha$, the corresponding boundary condition (32) is omitted. The proofs given here are easily modified along the lines of the discussion in this section so that duality results hold.

If $(P)$ and $\left(D_{0}\right)$ or $\left(P_{1}\right)$ and $\left(D_{1}\right)$ are independent of $t$, they reduce to the dual mathematical programming problems

$$
\begin{array}{ll}
\min f(x), & \text { subject to } g(x) \leq 0 \\
\max f(u), & \text { subject to } f_{u}(u)+y^{\prime} g_{u}(u)=0, y^{\prime} g(u) \geq 0, y \geq 0
\end{array}
$$

first considered by Mond and Weir [11].

\section{References}

[1] C. R. Bector, S. Chandra and I. Husain, "Generalized Concavity and Duality in Continuous Programming", Utilitas Mathematica 25 (1984) 171-190.

[2] A. Ben-Israel and B. Mond, "What is Invexity?" J. Austral. Math. Soc. Ser. B 28 (1986) $1-9$.

[3] S. Chandra, B. D. Craven and I. Husain, "A Class of Nondifferentiable Continuous Programming Problems", J. Math. Anal. Appl. 107 (1985) 122-131.

[4] B. D. Craven, "Invex Functions and Constrained Local Minima", Bull. Austral. Math. Soc. 24 (1981) 357-366.

[5] B. D. Craven and B. B. Glover, "Invex Functions and Duality", J. Austral. Math. Soc. Ser. A 39 (1985) 1-20.

[6] M. A. Hanson, “On Sufficiency of the Kuhn-Tucker Conditions”, J. Math. Anal. Appl. 80 (1981) 545-550. 
[7] O. L. Mangasarian, Nonlinear Programming (McGraw-Hill, New York, 1969).

[8] B. Mond, S. Chandra and I. Husain, "Duality for Variational Problems with Invexity", $J$. Math. Anal. Appl. 134 (1988) 322-328.

[9] B. Mond and M. A. Hanson, "On Duality with Generalized Convexity", Math. Operationsforsch. Statist. Ser. Optim. 15 (1984) 313-317.

[10] B. Mond and M. A. Hanson, "Duality for Variational Problems", J. Math. Anal. Appl. 18 (1967) 355-364.

[11] B. Mond and T. Weir, "Generalized concavity and duality" in Generalized concavity in optimization and economics (eds. S. Schaible and W. T. Ziemba), (Academic Press, 1981) 263-279.

[12] F. A. Valentine, "The problem of Lagrange with differential inequalities as added side conditions", in Contributions to the calculus of variations 1933-37, (University of Chicago Press, 1937) 407-448. 